\title{
Smart Prepaid Energy Meter based on Internet of Things
}

\author{
Mayur Rawte \\ Student, Department of \\ Information Technology, \\ Oriental Institute of Science and \\ Technology, Bhopal, India
}

\author{
Shrishti Sharma \\ Student, Department of \\ Information Technology, \\ Oriental Institute of Science and \\ Technology, Bhopal, India
}

\author{
Praveen Lalwani \\ Lit (ISM), Dhanbad, India
}

\begin{abstract}
As the advancement of technology and dependencies over the electrical appliances increases, the usage and requirement of electricity is also increases rapidly. Eliot is a smart energy prepaid meter based on Internet of Things ${ }^{[1]}$. This smart meter [2] will be helpful in generating the real-time data of the electricity usage. Users will be able to see their daily usage from a web app with the help of their unique identification number and password provided to them at the time of installation of this device. The main aim of this paper is to make a novel system which will help in reducing the usage of electricity and bring transparency between the electricity providers and the customers. This meter needs to be recharged similar to a mobile phone, payment for recharge can be done on a web portal designed for it. User will then be able to view the usage of electricity in a statistical manner with the balance available in their device. User can also stop flow of electricity from remote location if the electricity is not in use.
\end{abstract}

\section{Keywords}

Smart Energy Meter, Internet of Things, Prepaid Electricity Meter, NodeMCU, MQTT

\section{INTRODUCTION}

The Internet of Things (IOT) is defined by ITU and IERC as a dynamic global network infrastructure with self-configuring capabilities based on standard and interoperable communication protocols. In which, physical and virtual "things" have identities, physical attributes and virtual personalities, use intelligent interfaces and are seamlessly integrated into the information network. IOT is the network of physical objects like devices, vehicles, buildings, and other items embedded with electronics, software, sensors and network connectivity. This enables these objects to collect and exchange data. The internet of things allows objects to be sensed and controlled remotely across existing network infrastructure.

According to Gartner, 260 million objects will have their unique identity on the internet by year $2020^{[3]}$. We have quite seen that in our country, electricity billing system includes a lot of human intervention. In current scenario, meter readers manually take the meter reading by going home-to-home and note down it. These readings are forwarded to utility administration office. By evaluating these readings, electricity bills are generated. This scenario consumes a lot of time and energy.

Prepaid meters are the electronic devices that operate only when it has sufficient amount of balance. It records consumption of electric energy in real time. It has limitation like need of regular visit to recharge. Topping up at a local newsagent, petrol station or supermarket is inconvenient. Eliot is the smart meter which inherits the concepts of technologies like prepaid meters and Internet of things.

\section{LITERATURE SURVEY}

Friedemann Mattern and Christian Floerkemeier [1] discusses the vision, the challenges, possible usage scenarios and technological buiding blocks of "Internet of things". They conclude with the discussion of social and governance issues that are likely to arise as the vision of the Internet of Things to become reality.

S.Senthil Arumugam and S.Prabakaran [13] presents a understanding survey of smart electricity meters and their usage focusing on an understanding part of the metering process, distinct stakeholders interests, and the technologies utilized to meet the expectations of stakeholder interests. Besides they provide an outstanding part of challenges as well as opportunities arising exactly to the advent of big data and the accumulating well known of cloud environments.

Praveen Vadda and Sreerama Murthy [8] proposed a research work to measure and analyse the power consumption of electricity used by the customer using Smart metering. They use ARIMA model with XLASTAT tool with flattening technique for this purpose.

Azfar Tufail and Hummayoun Naeem [14] provide some enhancement in the conventional Metering system by smart metering. The term Smart Meter is an advanced energy meter that measures consumption of electrical energy providing additional information compared to a conventional energy meter.

\section{CURRENT SCENARIO AND PROBLEM DESCRIPTION}

Distribution and maintenance of electricity is owned by local state electricity board. Electricity usage of a user is calculated by calculating KWH electricity used over the period of a month. This reading is stored locally on the meter. A worker from the electricity board takes this reading by visiting doorto-door and manually notes it. This data is then forwarded to the head electricity board for evaluation, after evaluation bill is generated on the basis of the readings taken on monthly basis. These generated bills are again sent door-to-door by members of workforce. Then the recipients pay their bills by their favourable payment option. This process consumes a lot of time and human efforts and bill is totally dependent on the reading of the workers. So, whatever reading for a customer the worker takes, customer needs to pay for it and because of the post-paid method of payment for the electricity many users use electricity in very inefficient manner and sometimes they don't even pay for months. This results in loss to the electricity board and loss of electrical energy as well.

\section{PROPOSED WORK}

In the proposed scenario, Smart Energy has been emphasized which is an important conceptual paradigm for future energy use. Smart Metering involves installation of one or several 
Smart Meters by continuously monitoring and sending feedback of data to the customers. We will have a device called Eliot connected to the electric meter. This device will be installed by electricity board. At the time of installation of this device, staff will generate a unique identification number for device. Staff will then embed this number into the device and will also create a user account for the customer containing customer name, email, mobile number and address. The newly created device and user will be linked together. After successful installation of device and verification of the user details, customer will be provided with a password for checking status of their device with the help of email provided by the customer. Now the device is ready to be used. User needs to recharge this device from a web app designed for this device in order to continue supply of electricity. If the balance of this device becomes zero, this will stop the supply of the electricity after 24 hours, within that 24-hour user will get notification regarding balance status of the meter after a definite interval until he pays bill.

Electricity usage of customer will be sent to the server after a set time interval. Customer will then be able to log into their account from the web app and will be able to check their realtime electricity usage. This system aims to bring transparency of billing and usage between the customer and the electricity board. Customer will use less amount of electricity because of the prepaid nature of the billing system. Because of the psychological fact that if we pay for a resource before actually using it, we end up using less amount of resource as compared to post-paid method of payment. As a result, all the customer bill accounts will be cleared at the time.

\section{BENEFITS}

Eliot has many advantages over current scenario. These are as follows: -

- $\quad$ Easy attachment - We can easily set up Eliot anywhere as it is wireless and easy to install.

- Non-intrusive - Eliot does not cause any disruption because of its efficient working.

- Same Power - It measures same power consumption as typical revenue meter and also provides accurate billing.

- Confidentiality of sensitive data.

- Authenticity of data and precision in communication with target device.

- Supporting future expansion.

- Pay as you go -

1) Control your energy: - Set up balance related information to let you know when you're running out of balance.

2) Recharge anytime: - use Smartphone, App or our Website.

3) Track Energy Usage: - Eliot allows you to see how much energy you're using and also able to check their real-time electricity usage.

\section{WORKING MODEL}

Working of this device is based on the technologies like prepaid meter and Internet of things. This meter will be connected to NODEMCU (A System on Chip Wi-Fi module). This NODEMCU will be connected to local Wi-Fi access point. Every NODEMCU will have a unique identification number, at the time of installation of this device, staff of the electricity provider will assign this device identity to user of the that meter. This device needs to be recharged with some amount of money through the web portal of this device. This device will measure the electricity units on the blinking pulse rate of the LED of the meter and only allow the electricity to pass through if balance of that device is not zero. This device will be connected to a server through MQTT protocol (Message Query Telemetry Protocol). NODEMCU will be programmed such that it will send usage hourly to the server. Server will store all the incoming messages in database. This device will be connected to the electric meter via a circuit that will connect a GPIO pin of this device to the kwh pulse LED. Usage of electricity will be calculated on the basis of the KWH PULSE LED blinking. Each 1000 times a LED blinks 1 unit of electricity will be added to the usage and cost of that 1 unit will be deducted from the device, which will be updated onto the server in real time with the help of MQTT protocol. In order to have successfully transition of data a working internet connectivity is required via $\mathrm{Wi}-\mathrm{Fi}$ access point. If no internet connection is available at the time of operation data will be stored locally and will transmit the data whenever internet connection will be available.

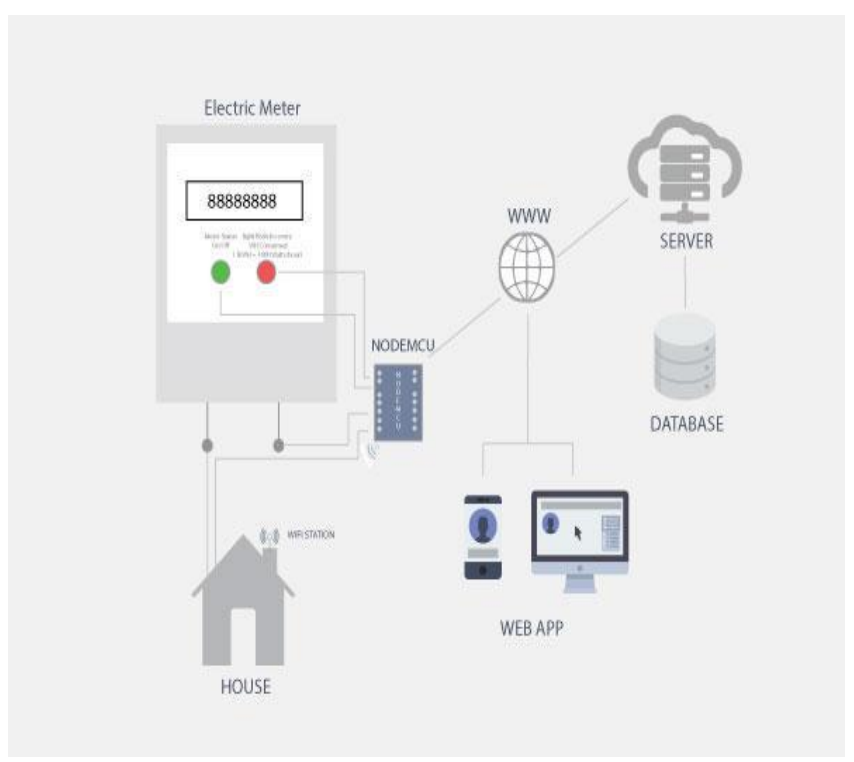

Fig 1. Architecture of Smart Energy meter based on internet of things

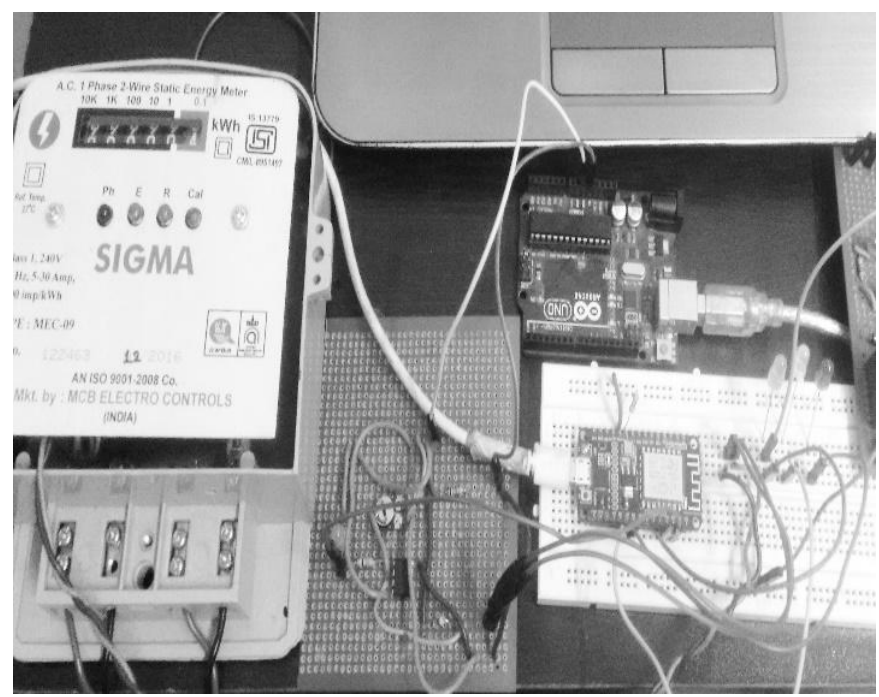

Fig 2. Working Model 


\subsection{NODEMCU}

NODEMCU ${ }^{[4]}$ is a Lua based ESP8266-E12 Wi-Fi SOC (System on Chip) module used commonly for the implementation of internet of things. It has 16 GPIO (General Purpose Input Output) and works on $3.3 \mathrm{v}$. The term "NodeMCU" by default refers to the firmware rather than the dev kits. The firmware uses the Lua scripting language.

\subsection{LUA}

Lua, a language for extending applications. Lua combines procedural features with powerful data description facilities, by using a simple, yet powerful, mechanism of tables. This mechanism implements the concepts of records, arrays, and recursive data types (pointers), and adds some object-oriented facilities, such as methods with dynamic dispatching. Lua presents a mechanism of fallbacks that allows programmers to extend the semantics of the language in some unconventional ways. As a noteworthy example, fallbacks allow the user to add different kinds of inheritance to the language. Currently, Lua is being extensively used in production for several tasks, including user configuration, general-purpose data-entry, description of user interfaces, storage of structured graphical metafiles, and generic attribute configuration for finite element meshes.

\subsection{MQTT}

The Internet of Things (IoT) has recently gained massive traction. IoT challenges enterprises, small companies, and developers with new problems to solve. While HTTP is the de-facto protocol for the human web, communication between machines at scale requires a paradigm shift - steering away from request/response and leading towards publish/subscribe. This is where the ultra-lightweight, massively scalable, and easy-to-implement protocol MQTT enters the picture.

MQTT ${ }^{[5]}$ (Message Queue Telemetry Transport) is a binary client-server publish/subscribe messaging transport protocol, standardized by OASIS. It is lightweight, open, simple, and easy to implement. Designed with a minimal protocol overhead, this protocol is a good choice for a variety of Machine-to-Machine (M2M) and Internet of Things applications, especially where a small code footprint is required and/or network bandwidth is at a premium. MQTT utilizes many characteristics of the TCP transport, so the minimum requirement for using MQTT is a working TCP stack, which is now available for even the smallest microcontrollers.

\subsection{DJANGO}

Django ${ }^{[6]}$ is a free and open-source web framework, written in Python, which follows the model-view-template (MVT) architectural pattern. It is maintained by the Django Software Foundation(DSF), an independent organization established as a 501(c)(3) non-profit.

Django's primary goal is to ease the creation of complex, database-driven websites. Django emphasizes reusability and "pluggability" of components, rapid development, and the principle of don't repeat yourself. Python is used throughout, even for settings files and data models. Django also provides an optional administrative create, read, update and delete interface that is generated dynamically through introspection and configured via admin models.

Some well-known sites that use Django include the Public Broadcasting Service, Pinterest, Instagram, Mozilla, The Washington Times, Disqus, Bit bucket, and Next-door.

\section{OPERATION}

The most common unit of measurement on the electricity meter is the kilowatt hour $[k W h]$, which is equal to the amount of energy used by a load of one kilowatt over a period of one hour, or 3,600,000 joules. Some electricity companies use the SI mega joule instead.

Demand is normally measured in watts, but averaged over a period, most often a quarter- or half-hour.

Reactive power is measured in "thousands of volt-ampere reactive-hours", (kvarh). By convention, a "lagging" or inductive load, such as a motor, will have positive reactive power. A "leading", or capacitive load, will have negative reactive power.

Volt-amperes measures all power passed through a distribution network, including reactive and actual. This is equal to the product of root-mean-square volts and amperes.

Distortion of the electric current by loads is measured in several ways. Power factor is the ratio of resistive (or real) power to volt-amperes. A capacitive load has a leading power factor, and an inductive load has a lagging power factor. A purely resistive load (such as a filament lamp, heater or kettle) exhibits a power factor of 1 . Current harmonics are a measure of distortion of the wave form. For example, electronic loads such as computer power supplies draw their current at the voltage peak to fill their internal storage elements. This can lead to a significant voltage drop near the supply voltage peak which shows as a flattening of the voltage waveform. This flattening causes odd harmonics which are not permissible if they exceed specific limits, as they are not only wasteful, but may interfere with the operation of other equipment. Harmonic emissions are mandated by law in EU and other countries to fall within specified limits.

We calculate the power consumption with the help of LED which flashes. The rate of LED flash is directly proportional to the amount of power passing through the meter. The Power can be calculated as follows: -

Power $(\mathrm{kW})=3600$ (seconds in 1 hour) divided by (the seconds between flashes*number of $\mathrm{Imp} / \mathrm{kWh}$ printed on meter)

For example, for the meter label states $1000 \mathrm{Imp} / \mathrm{kWh}$. If a constant $1000 \mathrm{~W}$ is going through the meter, then in one hour (3600 seconds) the Led will flash 1000 times, so the LED will flash after every $3600 / 1000=3.6$ second. 


\section{RESULT ANALYSIS}

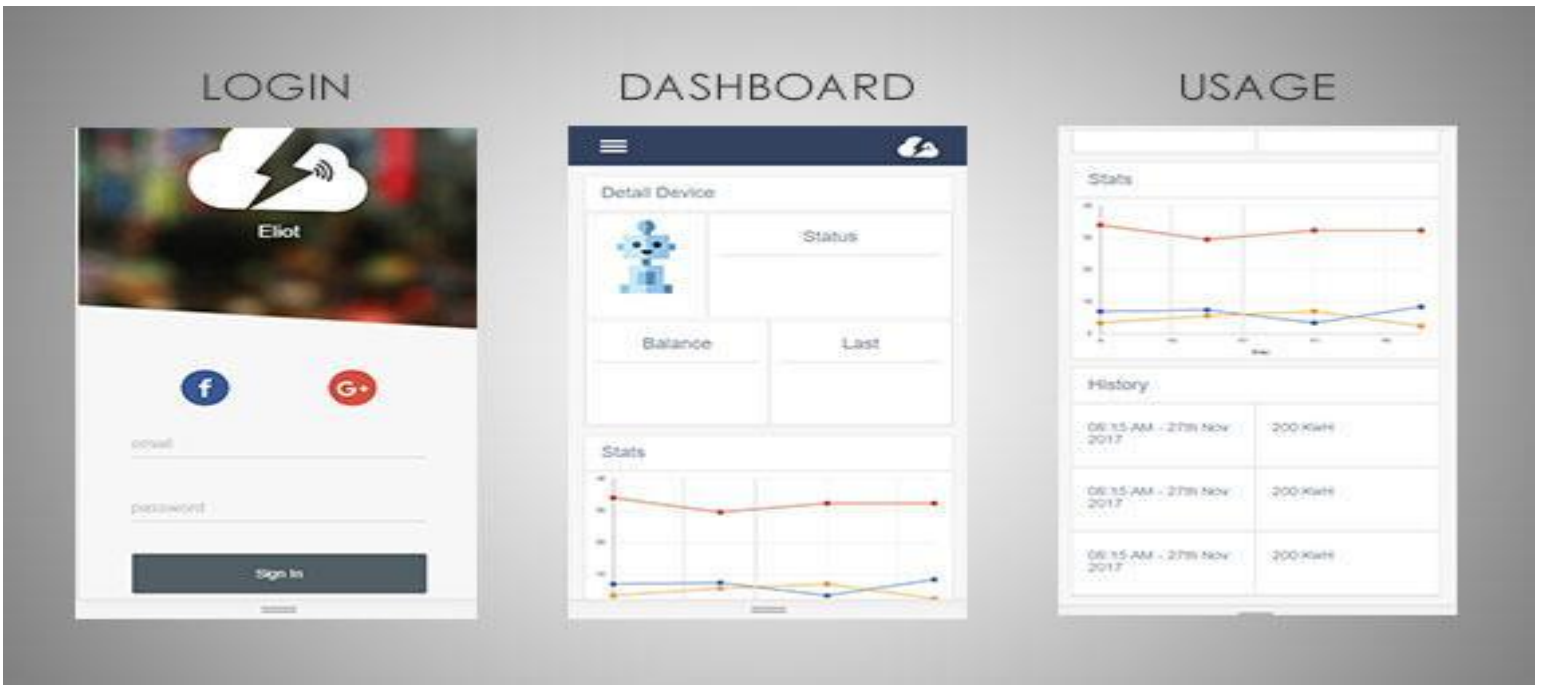

Fig 3. Eliot Mobile Application

\section{CONCLUSION}

With the increasing dependencies over electricity and internet, smart energy meter can be developed. These meters along with Eliot using the concept of prepaid meter and Internet of things can be generated. Eliot has been developed using wireless technology 802.11. It can also be replaced by Ethernet or 802.15 ZigBee Technologies. This smart energy meters can be developed to solve many problems such as over usage of electricity, large amount of manpower transparency of usage and wastage of money and resources etc. This technology allows verified customers to check status of electricity usage by using Device identification number and password in real time. This can be done from web application using Internet.

\section{FUTURE ENHANCEMENTS}

In the present system, Eliot smart energy meter help consumers to avoid unwanted use of electricity. The performance of the system can be enhanced by connecting all household appliances to IOT.

We can achieve following objectives to save power and avoid thefts:

- We can make an IOT system where a user can receive SMS, when he/she crosses threshold of electricity usage slab.

- We can make a system which can send SMS to the concerned meter man of that area when theft detected at consumer end

\section{REFERENCES}

[1] Mattern, Friedemann; Floerkemeier, Christian. "From the Internet of Computers to the Internet of Things". ETH Zurich. Retrieved 23 October 2016.

[2] DamindaAlahakoon and Xinghuo Yu. "Smart Electricity Meter Data Intelligence for Future Energy Systems: A Survey". (January 2015) in IEEE.

[3] Ovidiovermesan, Peter Friess. "Internet of Things- From Research and Innovation to Market Deployment".
[4] Vowstar. "NodeMCU Devkit". Github. NodeMCU Team. Retrieved 2 April 2015

[5] ISO/IEC 20922:2016 Message Queuing Telemetry Transport v3.1.1 https://www.iso.org/standard/ 69466.html

[6] Django FAQ https://docs.djangoproject.com/en/1.11/ faq/general/\#why-does-this-project-exist

[7] DarshanIyer N, Dr. K A RadhkrishnaRao." IOT based Electricity Energy Meter Reading, Theft Detection and Disconnection using PLC modem and Power optimization. (7, July 2015)

[8] Praveen Vadda, Sreerama Murthy Seelam." Smart Metering for Smart Electricity Consumption". (May 2013)

[9] E.F. Livgard," Electricity customers attitudes towards SmarrtMetering"in IEEE (July 2010)

[10] Z. Qiu, G. Deconinck, "Smart Meter's feedback and the potential for energy savings in household sector: A survey" in IEEE (April 2011)

[11] Andrea Zanella, senior member, IEEE, Nicola Bui, Angelo Castellani, Lorenzo Vangelista, Senior member IEEE, and Michele Zorzi, Fellow IEEE" Internet of Things for Smart Cities" (February 2014)

[12] Poonam Borle, AnkhitaSaswadhar, Deepali Hiwarar, Rupali S Kali," Automatic Meter Reading or Electricity". International journal of Advanced Research in Electrical, Electronics and Instrumentation Engineering. (March 2013)

[13] S. Senthil Arumugam, S.Prabakaran. "A survey of future energy systems using smart electricity meters". International Journal of Advanced Engineering and Recent Technology. (November 2016)

[14] Tufail Azfar, Naeem Hummayoun. "Advance enegy meter for smart metering system: an operational perspective" IOSR journal of Electrical and Electronic Engineering. (June 2016). 